\title{
Empirical Study on Recognition of Spatial Patterns in Choropleth Maps Using Hexagonal Shaped Units
}

\author{
Izabela Karsznia $^{\text {a,*}}$, Izabela Gołębiowska ${ }^{\text {a }}$, Jolanta Korycka-Skorupa ${ }^{\text {a }}$, Tomasz Nowacki ${ }^{\text {a }}$ \\ ${ }^{a}$ Department of Geoinformatics, Cartography and Remote Sensing, Faculty of Geography and Regional \\ Studies, University of Warsaw, Poland; i.karsznia@uw.edu.pl, \\ i.golebiowska@uw.edu.pl,j.skorupa@uw.edu.pl,t.nowacki@uw.edu.pl \\ *i.karsznia@uw.edu.pl
}

Keywords: Enumeration Unit Size, Map Generalization, Choropleth Map, Pattern Recognition

\begin{abstract}
:
Optimal enumeration unit size (EUS) plays an essential role in choropleth map generalization. In thematic maps, an EUS that is too small (i.e. too small a generalization level) results in a dispersed image and a difficulty with perceiving characteristic elements in the data (Robinson et al. 1984). On the other hand, a generalization level that is too large, expressed in a choropleth map by an EUS that is too large, also causes those spatial patterns in the data to be lost as the resulting image is too general. The authors are in favour of rather smaller EUS (Bregt and Wopereis 1990, Robinson et al. 1995, Dent et al. 2009). Moreover, they suggest using units of equal size and similar shape (Robinson et al. 1995, Schiewe 2019). This recommendation is met in a choropleth map consisting of geometric units, for example, squares, triangles, and hexagons. Thus thoughtful consideration of the EUS in choropleth map design is important to ensure the correct communication of spatial patterns. However, the EUS size and its influence on pattern conveying in choropleth maps have not yet been the subject of in-depth empirical studies. This research aims to address this gap. We focused on the issue concerning whether the ability to recognize spatial patterns is related to the hexagonal EUS, defined by the number of pixels. The aim is to indicate the range of EUS, namely, at what point the upper and lower borders of the range where the spatial patterns start and end is visible and recognizable by users.
\end{abstract}

To address this problem, we conducted an empirical study with 488 users. The participants were students from the two last years of high school education. The group consisted of 268 women and 220 men, 55\% and 45\%, respectively. The average age was 17, and ages ranged between 15 and 20. Asked about using maps, $11 \%$ participants declared that they used them every day, $32 \%$ once a week, $21 \%$ once a month, $19 \%$ once every few months, $6 \%$ once a year, and $11 \%$ used maps less than once a year. Empirical data was collected at the end of the school year; therefore, most participants had already completed the majority of their courses, thus we believe that they had received training to build their knowledge and abilities similarly to most citizens who have completed this obligatory stage of education.

Choropleth maps with eight EUSs were compared to a symbol map. For this purpose, nine maps were designed. All maps presented the same phenomenon but with different levels of generalization, which was expressed by the EUS. The symbol map presented the distribution of the phenomenon in a discreet way and was considered to be a presentation of the raw data. The symbol map assumes that every symbol (every dot) represents one geographical object. Some authors name this kind of map as a dot map arguing that a dot map is the result of a nominal map of points (Kraak et al. 2020). A dot map may also be confused with a dot density map. Thus, we call this kind of map a symbol map. Based on the symbol map, eight choropleth maps using hexagonal shaped units and a range of EUSs were prepared. The hexagonal unit sizes were recalculated into pixels. They correspond to 26, 52, 104, 208, 416, 832, 1664 and 3328 pixels (Figure 1). One independent and three dependent variables were defined. The EUS was considered an independent variable. The dependent variables were: (1) answer time, (2) users' preference for particular EUS for solving the given task, and (3) the expected response. The expected response was applied due to the exploratory character of the tasks in this study. The majority of the tasks applied in this research were tasks where there was no specific correct answer. Instead, the most common answer was explored. Thus, in the case of the majority of tasks, the expected response metric was applied during data analysis.

The participants each solved seven tasks. Four tasks were open and three tasks were closed. Within the open questions, the users were asked to rank all choropleth map variants according to their similarity or dissimilarity to the reference symbol map. Next they were asked to compare the symbol map with a choropleth map having a particular EUS in order to find and draw, on both maps, the corresponding areas in which the presented phenomenon was of similar shape and density. Within the closed tasks, the users were to use a 5-steps Likert scale to show which choropleth map (among all eight tested) they would prefer to use to describe the main characteristics of the phenomenon presented on the symbol map. Further, they answered the question asking how well the choropleth maps with their different unit sizes reflect the main characteristics (spatial patterns) of the phenomenon presented on the symbol map. 


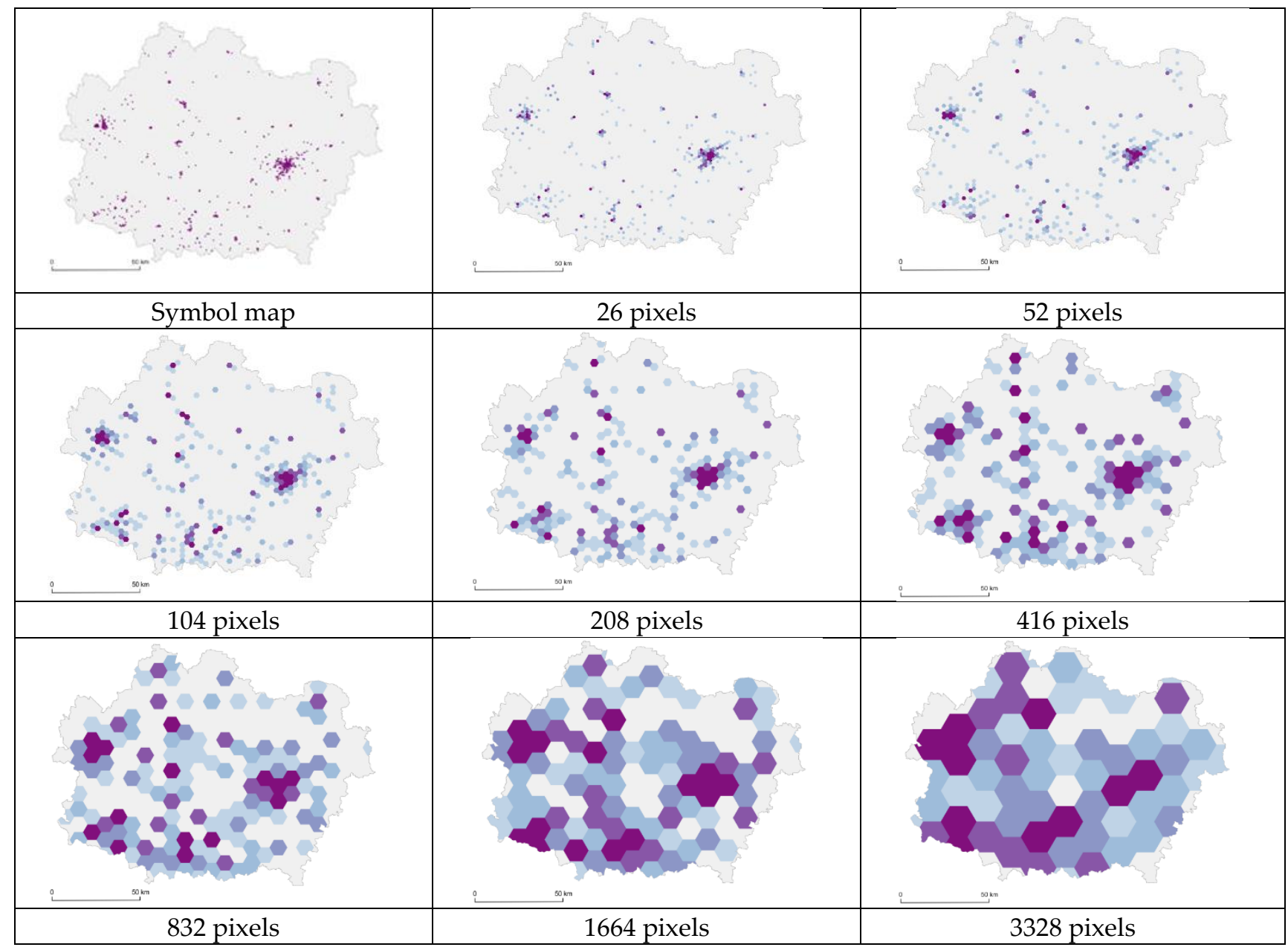

Figure 1. The maps used in the study with their corresponding applied EUSs.

The results show that the EUS has an impact on the users' spatial pattern recognition abilities. Choropleth maps with EUS of 26, 52, and 104 pixels were, in the majority, indicated by participants as those most suitable for indicating spatial patterns. This was in contrast to choropleth maps with EUS of 1664 and 3328 pixels, which users indicated as not being useful. However, there were some exceptions to this general finding. Thus, determining the optimal enumeration unit size is a challenging task, and requires further insightful investigations.

\section{Acknowledgements:}

This research was funded by the National Science Centre, Poland, grant number UMO-2016/23/B/HS6/03846, "Evaluation of cartographic presentation methods in the context of map perception and effectiveness of visual transmission". 